\title{
Mer, rivière, mangrove : les espaces marqués par le genre dans les communautés de pêche du Pará, Brésil
}

CHRISTINE ESCALLIER

\section{Introduction}

Les communautés de pêcheur, vivant sur les berges de l'estuaire de l'Amazone et du fleuve Rio Pará ${ }^{1}$, pratiquent leurs activités dans différents milieux : à l'embouchure où se rejoignent les eaux douces du fleuve et les eaux salées de l'océan Atlantique, dans les igarapés - bras très étroits et peu profonds des rivières de l'Amazone - et la mangrove. Ces milieux spécifiques engendrent une riche population ichtyologique, imposant des techniques de pêche et de navigation diversifiées, originales, adaptées aux différents écosystèmes. Pour atteindre ces pêcheries il faut autant naviguer que marcher.

La pêche étant la principale source de revenus des localités de la région dite du Salgado, dans l'État du Pará (Fig. 1), hommes, femmes et enfants participent au développement de cette économie. Il en découle une organisation sociale où les tâches effectuées, attribuées - exploitation de la ressource, activités pré et post-pêche - répondent à des règles strictes dominées par la vision du monde Homme/Milieu.

1 Le bassin du Rio Pará fait partie du bassin amazonien. Ils ont un estuaire commun. Le terrain concerne les villages de Abaetetuba, Cametá, Cachoeira de Arari, Colares, Igarapé Miri, Macapá de Barreta, Maracanã, Marudá, Mosqueiro, Pererú, Santa Luzia da Barreta et Vigia. 


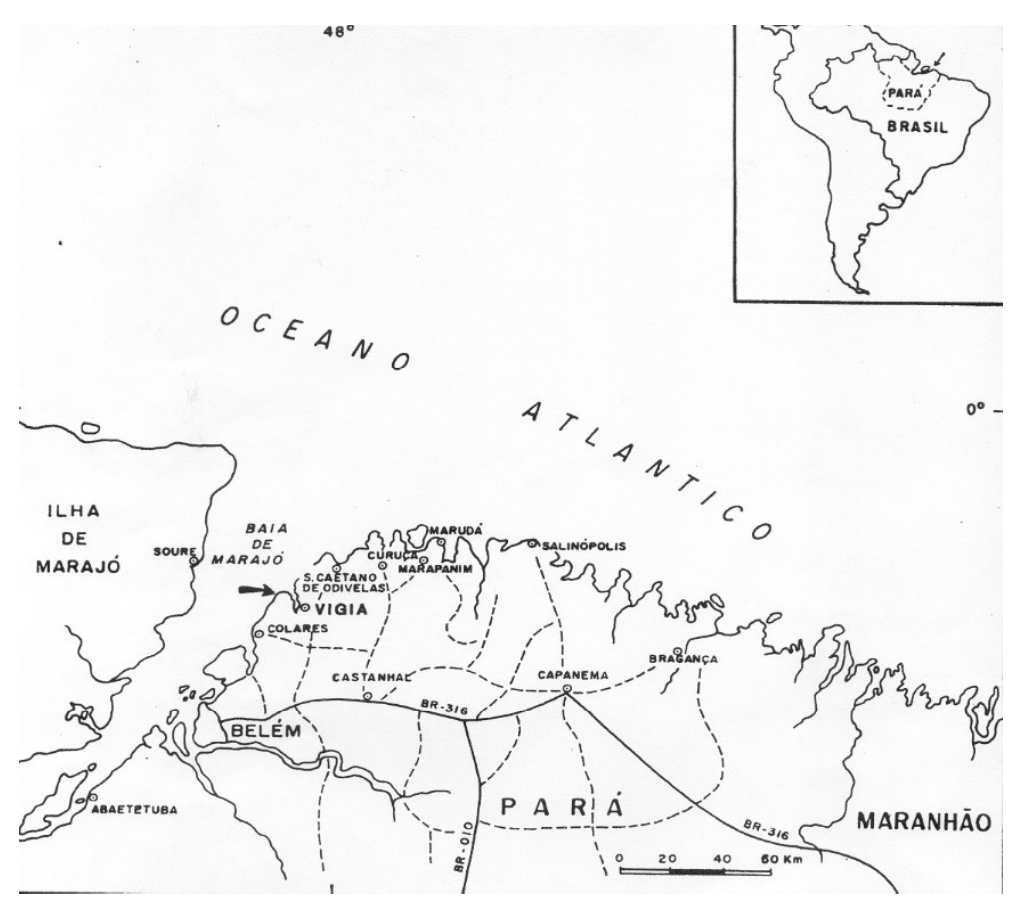

Fig. 1 - La zone du Salgado, État du Pará

La place des femmes dans l'univers marin s'est longtemps située dans un entre-deux monde invisible. Interdites d'embarquer, d'exercer la profession de marin-pêcheur, d'obtenir une cédula, l'équivalent du livret professionnel maritime, celles-ci se sont appropriées l'entre terre et mer : le rivage. Espace féminin par excellence, il est le lieu de l'attente du retour des hommes en campagne mais aussi celui du débarquement du produit de la pêche, de sa préparation (lavage, tri), de sa transformation (séchage, salage) et de sa commercialisation (mareyage, vende au détail), activités très souvent laissées aux mains des femmes. Elles jouent un rôle déterminant dans la réussite du métier exercé par les hommes. Leur apport méconnu à la vie économique et sociale des communautés de pêcheurs explique l'amplitude des études et des questionnements socio-anthropologiques portant aujourd'hui sur ces sociétés qui fonctionnent par dichotomie (Beck 1979 ; Godelier 1982 ; Geistdoerfer et alii, 1992 ; Woortmann 1992 ; Motta-Maués 1993, 1999 ; Diegues 1999 ; Frangoudes 2014 ; Escallier 2014). Pour la région paraense, on peut citer parmi les premiers travaux sur le rôle des femmes de pêcheurs ceux de la sociologue Cristina Maneschy (1995), de Edna Alencar (1991, 1993, 1997) et de l'anthropologue Lourdes Furtado $(1987,1993)$.

La reconnaissance légale du métier de pêcheuses dans de nombreux pays, et notamment au Brésil, peine à lutter contre les stéréotypes culturels - terreau des inégalités hommes-femmes -, les superstitions ou les interdits religieux.

\footnotetext{
«La province de Santa Catarina est située dans le sud du Brésil. [...] Dans cette région côtière, une source importante de revenus et de denrées alimentaires provient de la pêche, au moyen de petits bateaux et du système de l'économie familiale. En dépit de la pertinence du travail des femmes dans ce type de pêche, elles ne sont pas considérées comme des professionnels. C'est parce que la pếche artisanale
} 
est une modalité professionnelle réglementée par des normes qui n’offrent pas encore la même considération du sexe dans l'activité. (Boshi et al. 2012)

Dans ces communautés artisanales, toutes les femmes pêchent mais celles-ci doivent respecter des règles non-écrites, imposées par les hommes qui leur dictent à la fois en quel lieu elles peuvent s'aventurer et avec quel type d'engin de capture, limitant drastiquement leur champ d'action.

Il s'agit donc d'interroger le rapport homme/femme dans sa diversité des formes d'appropriation de l'espace aquatique et semi-aquatique, ainsi que le mode de production des espaces (Lefebvre 1974) qui s'opère dans les sociétés halieutiques suivant les genres (les épistémologies, usages et dispositions physiques distinctes qui en résultent) et d'introduire le concept de « mer sexuée », de ce qu'il engage tant au niveau des représentations, des pratiques sociales que des répartitions d'activités.

\begin{abstract}
« L'intérêt pour la dimension spatiale des phénomènes sociaux connaît actuellement un renouveau dans les sciences sociales, si bien que des auteurs, des historiens notamment, parlent de « tournant spatial » pour désigner ce nouveau regard [...]. Attachant une importance renouvelée aux catégories spatiales et aux spatialités, les travaux sur les femmes et le genre s'inscrivent dans cette tendance. Ce tournant spatial se manifeste à travers le recours aux notions de distance, de lieu, de paysage, d'espace et de territoire, mais aussi l'usage de dispositifs et de grilles de lectures spécifiques pour comprendre la spatialité des phénomènes sociaux, tels que l'approche multi-scalaire ou transcalaire. » (Direnberger \& Schmoll 2014:s.p.)
\end{abstract}

Les interdictions faites aux femmes, vivant dans la région estuarienne et les littoraux voisins, sont de pêcher en mer, d'embarquer sur un certain type d'unité, de ramender, trois activités qui s'exercent principalement dans le dehors, « au grand air ». Leurs actions sont également limitées à des espaces aux frontières visibles et tangibles comme celles des igarapés, semi-terrestres comme la mangrove ou terrestre comme leur domicile. Ces proscriptions précisent leur statut tant social que professionnel au sein de leur groupe. L'espace émerge alors dans sa dimension de quotidienneté en tant que produit culturel spécifique (Hall 1971) et éminemment politique.

Bien que la notion de pêche artisanale ${ }^{3}$ soit bien établie dans de nombreuses communautés halieutiques de par le monde et reconnue par la loi brésilienne, les hommes des localités isolées étudiées, éloignées des grandes centres, nient le droit aux femmes qui pêchent d'acquérir un statut identique au leur parce qu'elles pratiquent leurs activités dans des lieux proches des habitations, sans embarquement obligatoire (dans la mangrove) ou à bord d'unité mue par des rames, sans moteur, et pour une durée ré-

2 "La Provincia de Santa Catarina se encuentra en el Sur de Brasil. [...] En esta región litoral, una fuente importante de ingresos y alimentos de la población se deriva de la actividad pesquera, por medio de pequeñas embarcaciones y el sistema de economía familiar. A pesar de la relevancia del trabajo de las mujeres en este tipo de pesca, no son consideradas como profesionales. Esto es porque la pesca artesanal es una modalidad profesional regulada por normas que aún no ofrecen la misma consideración del género en la actividad."

3 Activité pratiquée par des familles de pêcheurs (par opposition à des sociétés commerciales), capturant des espèces en faible quantité à bord de petites unités ou à pied au cours de brèves sorties à proximités des rivages. 
duite à la journée. Les pêcheuses ne seraient donc pas des pêcheurs... La réalité montre le contraire. Et l'on vient à se demander comment une pêcherie - un espace technicisé à travers la capture du poisson - devient un espace « domestique » quand il est exploité par les femmes ?

\section{Une lutte de tous les instants}

La tâche quotidienne des femmes dans de nombreuses sociétés est d'œuvrer pour la survie de leur famille. Dans les communautés de pêcheurs, où les hommes sont absents durant plusieurs semaines ou rentrent bredouilles d'une énième campagne, cette survie est un dur et perpétuel combat. La rémunération à la part, qui caractérise les pêches artisanales, est à l'origine d'une profession aléatoire créant, dans bien des circonstances, une extrême pauvreté. Les petites communautés de pêcheurs artisans représentent, par ailleurs, l'une des classes socio-économiques parmi les plus déshéritées au niveau mondial. Particulièrement visible dans la zone du Salgado, j'ai pu également observer des conditions miséreuses sur différents terrains d'études dans les États du Maranhão, de Bahia et de Santa Catarina ${ }^{4}$.

«Les pauvres pêcheurs sont pauvres » ne cesse de répéter la FAO en publiant d'innombrables études pour la «Lutte contre la pauvreté dans les communautés de pêche artisanale $\gg(2002,2003, \ldots$ 2017) auxquelles l'OIT fait écho dans tous ses programmes visant à intensifier l>intégration de l>égalité entre hommes et femmes, y compris dans les programmes de promotion du travail décent et les politiques et stratégies nationales de réduction de la pauvreté.

« Dans les pays en développement, des millions de gens vivent au sein de communautés de pêche artisanale. On sait maintenant que tous les pêcheurs artisanaux ne peuvent pas être considérés comme pauvres, mais qu'une grande proportion d'entre eux le sont et le restent... [c'est l'auteur qui souligne]. Cette pauvreté persistante résulte de plusieurs facteurs dont l'origine se trouve à la fois à l'intérieur et hors du secteur halieutique [...] $\gg^{5}$ (FAO 2002)

Là où, selon la $\mathrm{FAO}, \ll[\ldots]$ il existe des poches de pauvreté où sont piégées des communautés de pêche, [...]. » (2003:70), les femmes doivent pallier l'absence d'argent et de vivres. Dans la nécessité impérieuse de se procurer l'indispensable, celles-ci ont mis au point différentes stratégies. Elles exercent de nombreuses activités de production et de commerce - toujours informelles - car les femmes de pêcheurs sont confrontées aux interdits sociaux, à l'immobilisme des idées et au pouvoir abusif des hommes qui les maintiennent dans une forme d'invisibilité (de - femmes cachées - selon les termes du président de la Commission parlementaire des droits de la femme et de l'égalité des genres ${ }^{6}$ ), en

$4 \quad$ Il s'agit principalement des villes et de leurs périphéries de São Luís do Maranhão, Ilhéus et Florianópolis.

5 Ces origines sont : la vulnérabilité ; l'insécurité de l'accès aux ressources ; la raréfaction des ressources ; l'isolement des communautés de pêche ; les mauvaises conditions socio-économiques, culturelles et politiques; un manque de soutien politique et financier ; l'analphabétisme...

6 “...there are what we can call "the hidden women"; Discours d'ouverture de l'audition publique - Developing the role of women in European Fisheries and Aquaculture - du 10 octobre 2013 au Parlement européen de BRuxelles. 
leur interdisant, notamment, d'exercer un emploi régulier et salarié qui leur imposerait de sortir de leur espace domestique. Seule Vigia échappe à cette interdiction, grâce à une petite manufacture de transformation du poisson qui embauche sporadiquement quelques femmes ${ }^{7}$. Les quelques postes à responsabilité sont entre les mains d'hommes. La méthode employée pour constituer un groupe de travail est casuelle et plutôt arbitraire. La veille d'une mise à quai importante, le directeur charge l'une des deux femmes employées de se rendre au domicile d'un certain nombre de collègues pour les informer qu'elles sont attendues à la fabrique le jour suivant. Sans liste préétablie d'employées temporaires, celle qui fait la tournée peut influer sur la composition du groupe et donc sur le revenu des familles. Sans contrat, les auxiliaires qui ont la chance d'être choisies acceptent des salaires dérisoires. Cet asservissement leur fait croire qu'elles seront appelées la fois suivante... Dans de plus petites communautés, comme São Caetano, les mairies et les écoles emploient des femmes pour faire le ménage dans leurs locaux. Les places sont rares et qu'elles déplaisent au maire et elles sont alors licenciées sans préavis, ni dédommagement. «J'ai travaillé 8 mois à la préfecture de São Caetano comme servante. Un nouveau préfet est arrivé et m'a licenciée pour mettre à ma place quelqu'un qu'il connaissait. Je me suis retrouvée à la rue. » (Telma, 28 ans, São Caetano). D’autres ont eu un temps l>espoir de trouver de l>embauche dans une affaire qui avait été implantée dans le village. Il $s>$ agissait $\mathrm{d}>$ un élevage de poulets qui employait sept personnes pour $1>$ abattage des volailles :

«Les propriétaires sont venus d'une autre région et ils ont installé leur élevage. On travaillait 3 jours par semaine, de 8 à 14 heures. Ça arrivait souvent qu'ils ne nous paient pas. Ils nous donnaient alors 10 aufs pour la journée. Dix aufs! J'avais 7 bouches à nourrir à la maison et par d'argent pour acheter la farine. Ça a duré comme ça un an et demi. Ils ont fermé, tout vendu et sont partis avec beaucoup d'argent. Et nous, on était toujours aussi pauvres. Ils le savent, les gens, alors ils en profitent. » (Idalina, 24 ans, São Caetano)

À Marudá, elles lavent le linge ou font la cuisine chez autrui ${ }^{8}$. Celles qui ont un réfrigérateur ce qui est rare - vendent de la glace ou des jus de fruits frais dans la rue, d'autres des bouillies de maïs. Parfois elles montent une petite entreprise financée par un microcrédit permettant d'acquérir une carriole qui facilite un commerce itinérant ${ }^{9}$. Presque toutes cultivent quelques plantes aromatiques ou médicinales, souvent du manioc (Fig. 2), sur un lopin de terre qu'elles se partagent entre voisines. Très tôt, les filles de pêcheurs sont à la tâche et accompagnent leur mère dans toutes ces activités annexes.

Mais de toutes ces stratégies, c'est la pêche et ses activités parallèles qui organisent le quotidien des femmes. Leurs villages palustres, construits à la lisière de la mangrove, des igarapés et du fleuve, loin des autres municipalités, réduisent leur horizon et leur espace d'activité. Ces écosystèmes leur offrent

7 Lors de ma visite des lieux, seules deux femmes y étaient employées à l'année et une quarantaine d'autres pouvaient être appelées en renfort en cas d`un surcroit de travail.

8 En période estivale, les habitants de la capitale Belém viennent en villégiature à Marudá. Les femmes sont alors employées comme domestique dans ces résidences d'été.

9 Le microcrédit s'est peu à peu enraciné au Brésil et particulièrement dans les régions délaissées, offrant des taux d'intérêt plus bas que ceux pratiqués par les banques, $4 \%$ au lieu de $14 \%$ dans ce cas étudié. 
la source d'approvisionnement qui leur manque. Tandis que les hommes exploitent les pêcheries de l'estuaire de l'Amazone ou les côtes du Pará et de l'île Marajó (Fig. 3) durant deux à trois semaines, les femmes exercent des pêches dites «de proximité» sur les rivages et dans la mangrove - le « garde-manger » du pauvre -, la mer restant le territoire des hommes.

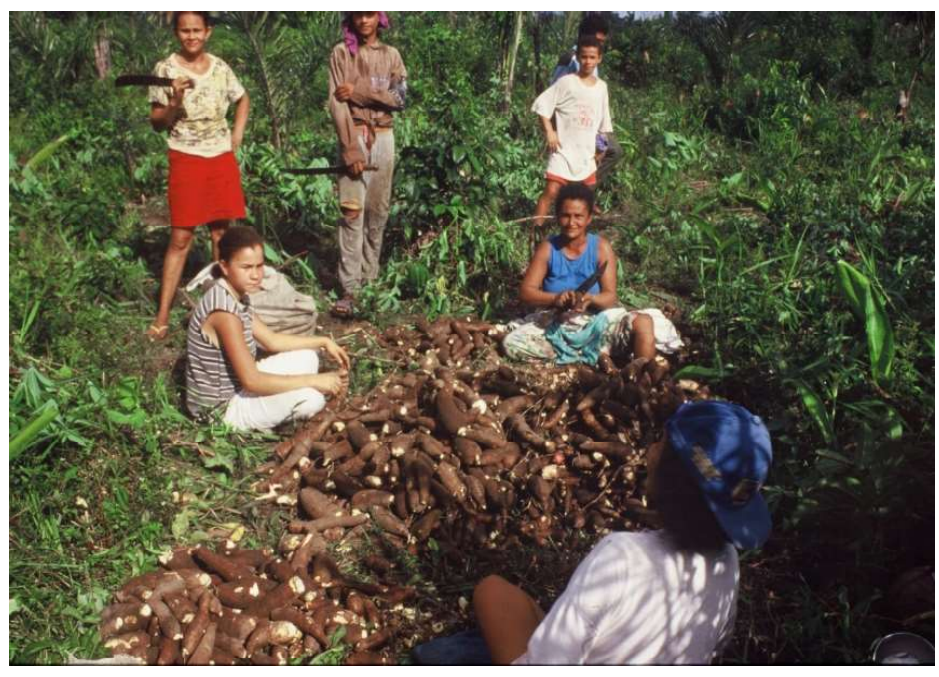

Fig. 2 - Femmes dans la forêt récoltant le manioc

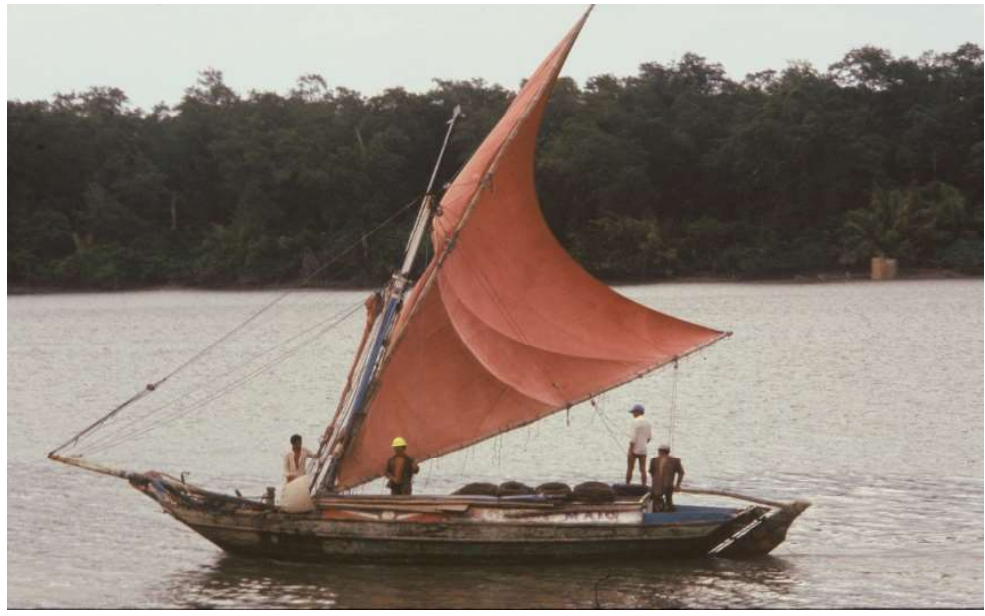

Fig. 3 - Barque de pêche dans l'estuaire de l'Amazone

Dans bien des localités les femmes récoltent les crustacés comme le mexilhão (variété de moule), la crevette dans le fleuve ou l'igarapé, le turu (sorte de larve) et le crabe caranguejo ou le siri-azul (crabe bleu, callinectes sapidus) dans la mangrove. La capture des crabes nécessite de la main-d'œuvre. Hommes, femmes et enfant, s'y emploient :

«Mon mari a une barque. On pêche en famille: mon mari et mes 7 enfants âgés de 6 à 30 ans, sauf ma mère qui est trop vieille. Nous allons à la baraque (cabane en bois construite dans la mangrove, N.d.A.) et on y reste des semaines entières. On tire au lacet les crabes, on revient et on vend. » (Regina, 49 ans, São Caetano) 
L'expression « tirer le crabe » (tirar o caranguejo) explicite la technique employée pour l'attraper. Caché dans la boue de la mangrove, souvent à une profondeur d'environ un mètre dans un terrier oblique dans lequel il s' accouple, le tireur ou la tireuse de crabe ${ }^{10}$ doit plonger littéralement le bras jusqu'au cou pour attraper le crustacé et l'extirper d'un substrat épais et visqueux. Cette technique harassante se pratique certains mois de l'année, quand le crabe reste caché. Quand il s'aventure hors de son refuse, en période de reproduction, les tireurs/tireuses posent un collet au-dessus du trou qui le piège au moment où il sort, ou le cueillent à même le sol. Mais il ne suffit pas de se baisser pour le prendre car l'animal est vif et court très vite entre les racines des palétuviers. Pliés en deux les tireurs tentent de les suivre mais il est difficile de courir dans la mélasse d'argile qui colle aux tongs et les avale au passage.

Les crabes sont vendus entiers et frais sur les marchés (Fig. 4). Les invendus du jour sont décortiqués : leur chair débarrassée de sa carcasse est mise en sachet et conservée :

« Quand je n'ai pas vendu les crabes le jour même qu'ils ont été tirés de la mangrove, ma fermme en extrait la chair. Ça donne du travail mais ça rapporte plus parce que 100 crabes, ça donne $3,5 \mathrm{~kg} d e$ chair ce qui font 28 reais au lieu de 10, parfois moins. II faut un jour, 4 à 5 heures pour les cuire dans l'eau, les ouvrir et ôter la chair. » (Antônio, tireur de crabes, 30 ans, São Caetano)

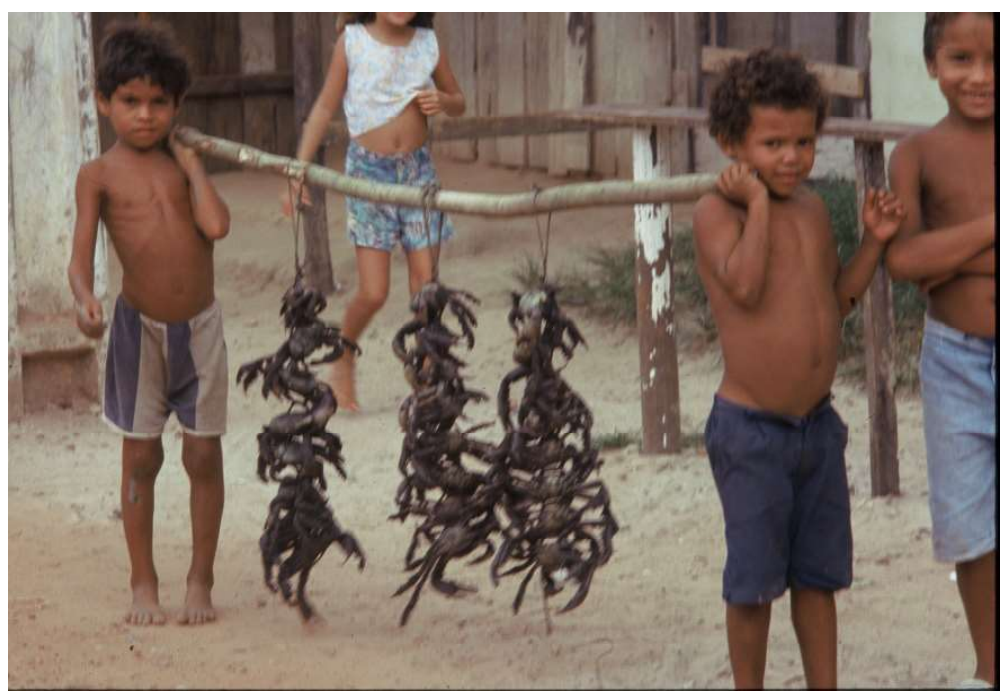

Fig. 4 - Enfants revenant de la mangrove avec des crabes

Cette transformation est une activité résolument féminine, encore mal organisée dans certaines localités parce que pratiquée à domicile, sans grand respect parfois des conditions d'hygiène. À São Caetano, la vente de la chair de crabe fait l'objet d'un commerce entre les producteurs locaux et les revendeurs de Belém, la capitale. Le village est réputé pour cette activité qui donne lieu à un Festival

10 Tirador/tiradora de caranguejo appelé aussi caranguejeiro ou carangueijera. 
chaque année en décembre ${ }^{11}$ (Fig. 5), considéré comme l'une des principales manifestations de valorisation de la gastronomie et de la culture locale.

De cette célébrité, les femmes de São Caetano ne gagnent ni en reconnaissance ni en visibilité. D'autant, quand elles pêchent/tirent entre elles, au quotidien, c'est rarement pour vendre le produit de leur travail. Elles pêchent pour le déjeuner ou le dîner. « Parfois quand il faut nourrir les enfants, je vais tirer quatre crabes pour manger. J'y vais avec la voisine ou une petite d'ici. » À leur retour, elles cuisinent et servent leurs enfants. Cette immédiateté dans l'action et le fait de souligner quelles font, en quelque sorte, « leurs courses, leur marché », comme une citadine irait au supermarché, renforce le discours tenu par les hommes qui est d'associer ces pratiques non pas à des activités professionnelles mais à de simples affairements domestiques.

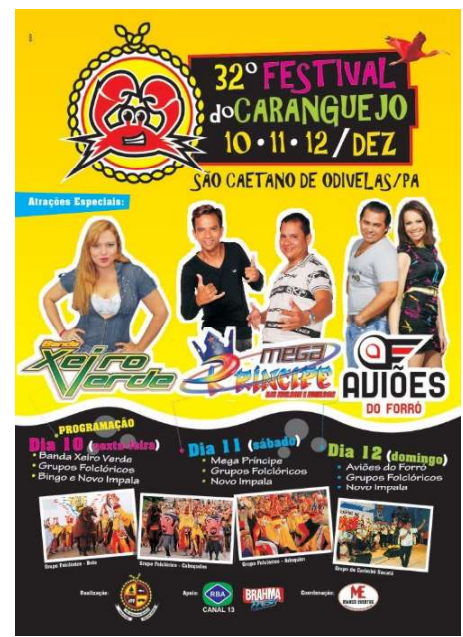

Fig. 5 - Festival du crabe à São Caetano (Auteur inconnu Crédit DR)

La plupart des femmes de ces localités vident les currais. Ces énormes pièges, postés le long du rivage (Fig. 6), sont montés avec des branches et des racines prises dans la mangrove, formant un enclos. Comme la récolte des crustacés, le curral est une activité propre aux petits pêcheurs qui s’associent pour son exploitation et qui nécessite tout au plus une simple petite barque pour y accéder et parfois des heures de marche sur l'estran. Quand les hommes sont en mer, il incombe aux femmes et aux enfants d'aller les vider (Fig. 7 et 8 ).

11 Ce festival aurait réuni près de 30.000 participants en 2014 selon le quotidien Globo. http://g1.globo.com/pa/para/noticia/2014/12/festival-do-caranguejo-deve-reunir-30-mil-em-sao-caetano-no-para.html 


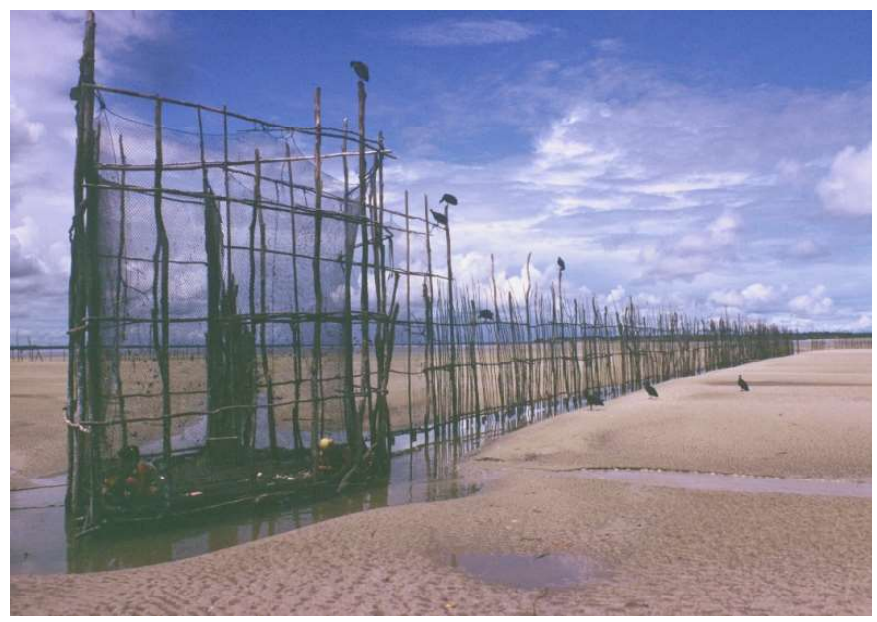

Fig. 6 - Curral à marée basse aux environs de Marudá
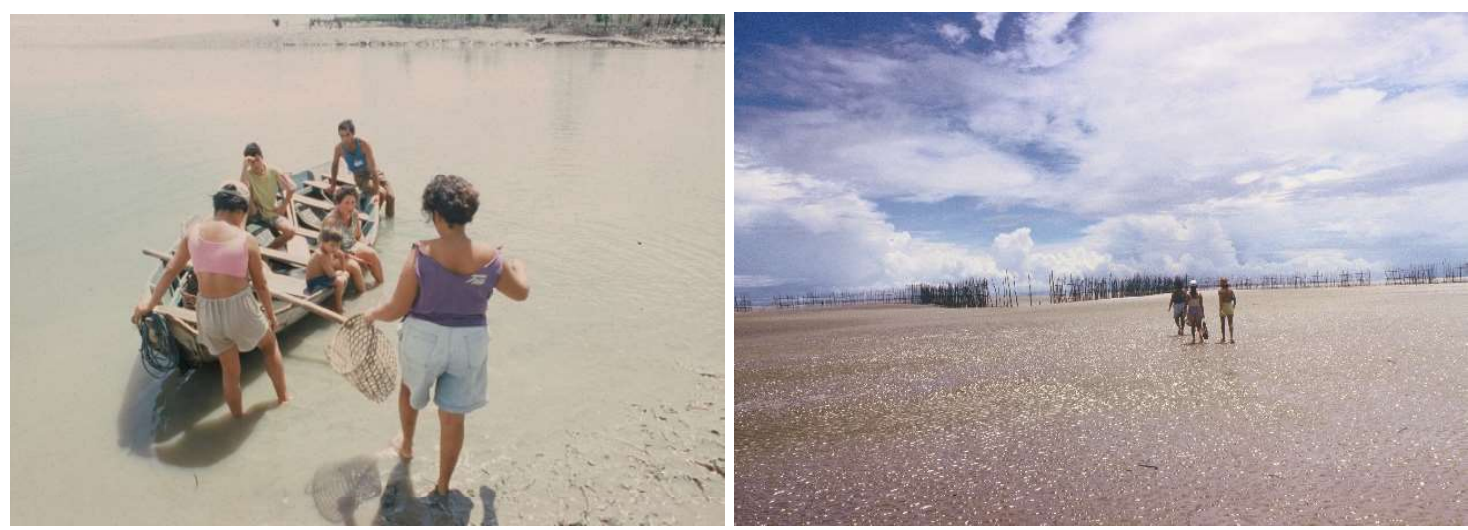

Fig. 7 - Femmes et enfants traversant l'igarapé pour atteindre la mangrove et marche vers le curral

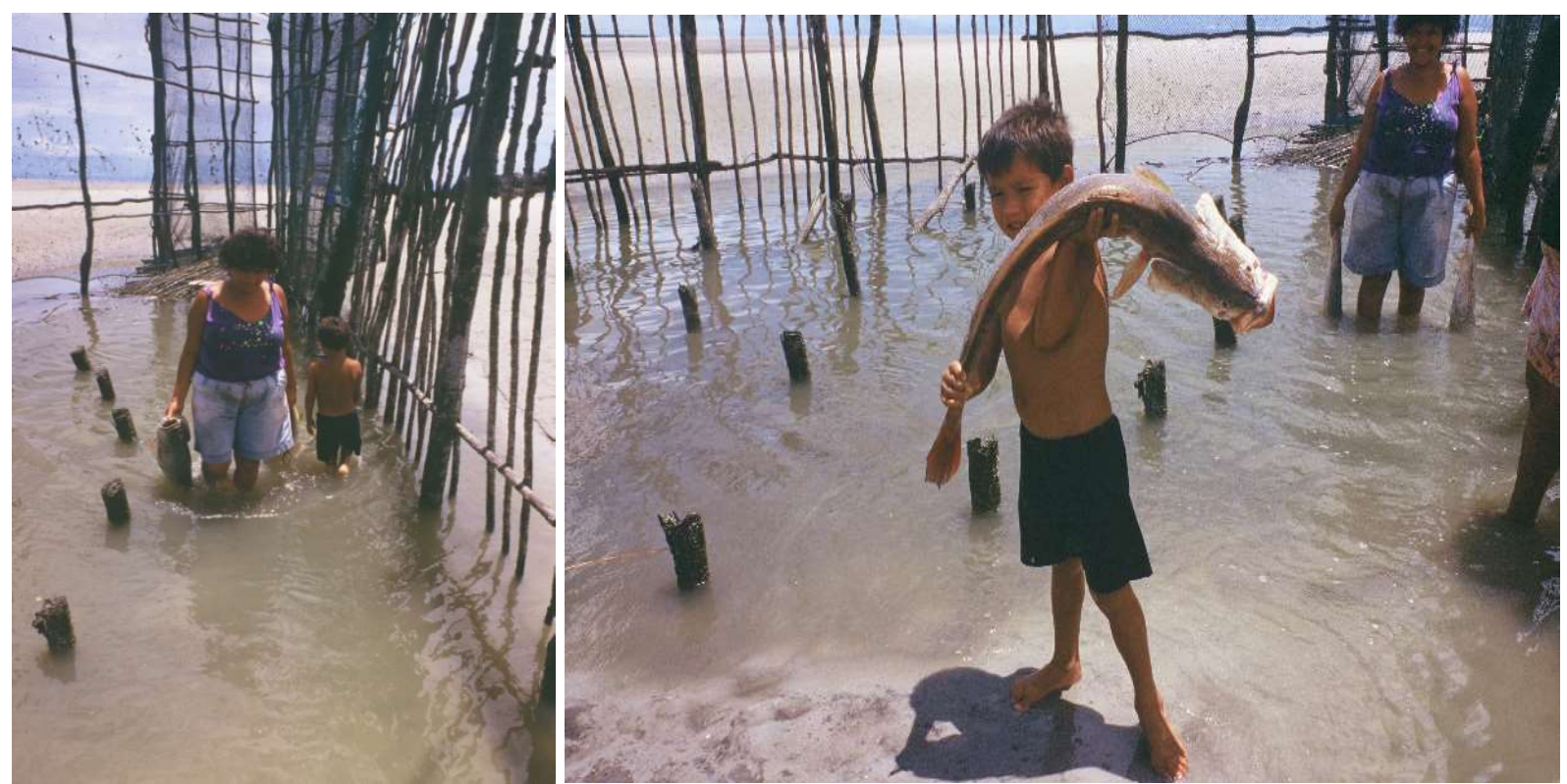

Fig. 8 - Femme et enfant au curral 
Les femmes emploient presque toujours des engins de captures de petites dimensions ou rudimentaires tels que le filet draguant puça, long pour capturer les crevettes et rond pour pêcher le crabe siri, des filets lancés comme l'épervier qu'elles jettent au-dessus des roches immergées à marée-basse, le lacet pour attraper les crabes dans la mangrove. Elles vont parfois pêcher seules mais la plupart du temps elles s'ont accompagnées d'un de leurs enfants ou partent en petit groupe qu'elles forment avec leurs voisines.

Pour manœuvrer des filets draguants en travers d'un igarapé ou d'un furo ${ }^{12}$, il faut au moins être deux. Travail pénible, il est souvent effectué par un pêcheur aidé de sa femme. «Je suis allée pêcher avec mon mari 5 jours de suite. On y va en barque, alors on traverse le fleuve et mon mari me laisse pour pêcher la crevette avec un filet de $300 \mathrm{~m}$ de long. » Après quelques coups de rame pour quitter la rive et mettre en position la barque en travers du cours, la femme se glisse dans l'eau pour positionner et fixer le filet. Immergée jusquà la poitrine ou jusqu’au cou, elle avance lentement, draguant le fleuve à pied et parcourant parfois de longues distances pour atteindre la pêcherie, tandis que l'homme, assis dans la barque, positionne l'engin : «Ce que je préfére c'est pêcher au filet. C'est plus divertissant car par exemple tirer le puça c'est fatiguant parce qu'on est dans la boue. Avec le filet, tu vas en barque quand la marée est basse, tu descends et tu prends un bain! » (Mundica, 49 ans, Marudá).

L'ensemble de ces activités amènent les femmes à quitter l'univers réducteur qu'est leur maison. Et malgré la dureté de la besogne, les conditions - la chaleur diurne, la moiteur, les insectes vecteurs de maladies... - qui épuisent les corps, toutes disent pourtant qu'elles « aiment pêcher » bien quelles n'en tirent aucune reconnaissance sociale ou professionnelle. Les occasions de sortir sont en fait rares et c>est pour cela que celles qui vont pêcher le font avec plaisir. «Tous les jours j'y vais. J'aime ça. J'y vais la nuit. Pour tout j'y vais : au turu, etc. J'étais enceinte de 8 mois que j'allais pêcher. », et certaines sans aucun bénéfice pécuniaire car : «Tout est bon pour sortir. J’aide souvent vider les currais mais je ne reçois rien en échange, ni argent, ni part de poissons. » (Socorro, 30 ans, Marudá). Mêmes les filles d'agriculteurs vont à la pêche. «Je vais au siri, en décembre, le samedi et le dimanche vers 5, 6 heures du matin. J'utilise un puça, et une barque qui appartient à mon père qui l'utilise pour planter le riz. Je pêche en face de Vigia. » Mais malgré ce discours enjoué, qui semble faire de la pêche un loisir, il ne faut pas oublier que ces sorties sont indispensables à l'équilibre économique et alimentaire des foyers. Quand le poisson est vendu, cet apport d'argent permet aux femmes d'acquérir certains produits industriels comme le café, le sel, le sucre et surtout la farine de manioc, base alimentaire et substitut quand le poisson vient à manquer, ce qui est fréquent.

«Mon mari a travailléplusieurs jours. II partait à l'aube et rentrait la nuit. À la fin de la semaine,
après avoir déduit les dépenses du canot et vendu le poisson, et bien il n'a rien tiré de ce travail; seu-
lement 50 centimes. Il a été courageux. Il a pêché presque 300 kilos de poissons à un real le kilo, moins
les dépenses et l'avance, il lui restait la moitié d'un real. Un kilo de farine coûte un real. » (Risoleida,
27 ans, Vigia)

12 En Amazonie brésilienne : communication naturelle entre deux fleuves ou entre un fleuve et un lac. 
La malnutrition est d'ailleurs la cause principale de l'inattention des enfants à suivre une scolarité et explique les nombreux échecs. La plupart partent à l'école le ventre vide ${ }^{13}$ :

«Ma fille revenait de l'école à 11 beures en disant «Ah maman, j'aifaim». Ah ma fille, je n'ai rien, je lui répondais, prend un chibé (eau mélangée à de la farine de manioc et du sucre, N.d.A.) ${ }^{14}$ - c'est très liquide et on le boit à la cuillère. Parfois ma fille insistait et je répondais «mais ma fille, je n'ai pas de farine». «Mais même ainsi» disait-elle, mais un chibé sans farine ce n'est plus que de l'eau. «Mais je veux un chibé' elle répétait, «je ne veux pas boire de l'eau». Elle ne comprend pas. Mon mari lui, sort sans rien manger. II boit seulement un petit café. II va pêcher ainsi. II lance le filet en haut (désignant la rivière), tire jusqu'en bas, hale le filet, et il n'y a rien. Quand il rentre en fin d'après-midi je dis «Où est l'argent pour acheter la farine?». Il n'y en a pas. Mais il doit aller pêcher, toujours... » (Dona Maria, São Caetano)

Et il n'est pas rare qu'une famille entière - père, mère, enfants, jusqu'au chien et aux quelques poulets faméliques -, se contente de farine et d'eau un jour entier.

Comme pour la plupart des femmes, la journée de pêche n'est qu'une partie des activités quotidiennes auxquelles il faut ajouter les tâches domestiques :

«J'ai un mari et deux fils qui vont à la pêche. J'ai en plus cinq enfants qui restent à la maison. Je dois laver les vêtements, je dois faire le repas de l'un, puis le repas de l'autre. Je me lève tôt. Un étudie, il doit arriver à 7 heures à l'école, je dois me lever à 6 h30. Je fais le café. Vers 4-5 heures de l'après-midi, ils rentrent tous, ils prennent un bain, je prépare le dîner. Mon mari revient de la mangrove en fin d'après-midi. C'est lui qui va au marché acheter de quoi manger pour les enfants. C'est une lutte jusqu'à 8 heures du soir. Et puis vient une autre «fournée», à 9 heures du soir. Ce sont ceux qui reviennent de l'école du soir. » (Maria, 47 ans, São Caetano)

Pêcheuses au quotidien ou occasionnelles, cet emploi du temps reste celui de la plupart des femmes. Cette lutte de tous les instants les pousse alors à enfreindre les règles sociales préétablies en empiétant sur les prérogatives des hommes qui sont notamment d'embarquer et de pêcher.

Cette grande vulnérabilité des femmes, observée sur tous les continents, a amené le Parlement Européen, en 2013, à réunir exceptionnellement deux Commissions - celle des pêches et celle des

13 Pour lutter contre l'absentéisme et l'analphabétisme des enfants qui doivent accompagner leur père à la pêche, l'école primaire de Marudá offre un déjeuner aux élèves après leur matinée de cours.

14 Terme d'origine tupi qui désigne une boisson d'Amazonie, sorte de bouillie faite d'eau et de farine de manioc parfois accompagnée de sucre, de sel, d'épices. 
droits de la femme et de l'égalité des genres ${ }^{15}$ - pour une Audition publique à Bruxelles réunissant des experts affirmant que « dans les communautés de pêche tout le monde sait qu'»une entreprise de pêche ou d'aquaculture a derrière elle une femme». » (Escallier 2013 ; Frangoudes 2017:25)

C'est pourquoi les femmes élèvent de plus en plus la voix afin d'exiger que leurs droits soient enfin reconnus.

\title{
Pêcher, une affaire d'homme...
}

Bien qu'il soit clairement établi que les pêches féminines soient avant tout un moyen de se procurer de la nourriture au quotidien, de remédier au manque d'argent et d'aider les hommes à assurer la survie du groupe, il n'en reste pas moins que ces derniers acceptent à contre cœur de voir les femmes lancer un filet ou haler une palangre. Pêcher est une affaire d'hommes. « Les hommes n'aiment pas que les fermmes pêchent. Ils les appellent des hommasses (machão ${ }^{16}$ ). Surtout à Soure (Ile de Marajó) les hommes ne veulent pas voir les femmes pêcher. Mais ailleurs aussi, à Tupinamba, etc., et ici c'est pareil. » (Cileusa, São Caetano).

De nombreux témoignages confirment combien il est difficile pour les femmes à la fois d'assurer l'équilibre économique familial tout en préservant leur image sexuelle stéréotypée, construction sociale du féminin évoquant une image de faiblesse et de soumission:

\begin{abstract}
«On m'appelle hommasse quand on me voit lancer l'épervier. Mais que peuvent faire les femmes pour subvenir aux besoins de leur famille et aider leur mari? Justement, c'est se qu'on se demande! Que peuvent faire les femmes? Les hommes pensent que pêcher c'est un travail d'homme. C'est mon mari qui m'a enseigné à pêcher. Quand les autres hommes l'ont vu, ob ils ont été en colère! Ils l'ont critiqué. » (Risoleida, 27 ans, Vigia)
\end{abstract}

Les insultes proférées par les hommes donnent mot et image à l'expression des préjugés : « On a dit que j'étais une pute parce que je pêchais avec une barque! » mais ceux qui acceptent d'emmener leur femme à la pêche sont tout autant objet d'une désapprobation collective.

«Mon mari et son frère sont associés. Ils ont un curral. Mon fils va avec eux. Parfois, il n'a personne pour y aller alors j'y vais. II y a des hommes qui nous voient et qui lui crient «Eh, tu vas tuer ta femme» ou encore «Tu aimes tellement ta femme que tu la laisses patauger dans la boue! «» (Maria, 32 ans, Marapanim)

15 L'audition publique «Developing the role of women in European Fisheries and Aquaculture » avait pour objet d'obtenir un aperçu clair des progrès escompté en ce qui concerne la participation des femmes dans le secteur de la pêche et de l'aquaculture. Les discussions portaient sur la situation présente, sur les perspectives futures de femmes dans les pêcheries européennes, les programmes du Fonds européen pour la pêche en faveur des femmes et aux réseaux et aux associations de femmes.

16 Machão terme péjoratif : " celle dont les traits, le son de la voix, la taille, les manières tiennent plus de l'homme que de la femme ». Définition du dictionnaire Domingos de Azevedo. 
«Il y a eu beaucoup de gens qui ont été contre le fait que je pêchais. Ils parlaient, parlaient, critiquaient. Maintenant non, parce qu'ils ne peuvent plus rien dire contre moi mais dès que j'ai commencé, au début, je passais près de leur barque et ils disaient à mon mari: «Eh, fils de pute, tu n'es pas un homme pour laisser pêcher ta femme! «. » (Raimonda, 53 ans, Marudá)

Dans ces communautés où le poids des traditions se fait sentir tant dans les propos que dans les attitudes, des femmes contribuent à la reproduction des inégalités et à la transmission des stéréotypes du genre :

«Tant que les femmes pêchent seules, ou accompagnées d'un fils, et avec des moyens rudimentaires, les autres femmes de la communauté trouvent ça normal. Il en va autrement si elles vont pêcher avec leur mari (en barque et avec un filet, N.D.A.). Celles qui restent toute la journée à la maison par obligation les jugent et les jalousent parfois. » (Neuza, 42 ans, Colares)

Toutes ne condamnent pas ouvertement leurs consœurs qui transgressent les règles. Certaines les intériorisent et se conduisent en conséquence c'est-à-dire selon les normes de comportement implicitement imposées par le groupe et rappellent que la pêche est une affaire d'homme :

«Ah, certaines fermmes disent «Tu ne fais pas confiance à ton mari c'est pour ça que tu le suis.» Elles disent «Tu es jeune, tu es belle et tu restes ainsi, tes jambes sont pleines de varices, et ci et ça.» Avec moi, beaucoup m'ont dit: «De la boue, de la boue, prendre le soleil... ton mari est jeune, tu as un compagnon poury aller à la pêche et tuy vas ?’ Les autres femmes ne comprennent pas car moi j'y vais parce que j'aime ça. Mon mari m'invite souvent à venir avec lui. Ces fermmes sont peut-être jalouses parce que leurs maris ne veulent pas les emmener, elles! Et elles aimeraient bien y aller aussi et sortir de chez elles. » (Dinoralina, 46 ans, Vigia)

Ces attitudes hostiles sont cautionnées par l'église locale, voire dictées par elle. Des articles parus dans le journal de Vigia - O Pescador ${ }^{17}$ - ont relaté un fait survenu dans la communauté : une jeune femme de 41 ans, Ângela (Fig.9), était veuve depuis peu. Son mari, pêcheur et armateur d'une embarcation à moteur de 10 tonneaux et d'un filet, avait été assassiné par l'un des hommes de l'équipage et son bateau volé.

17 Le Pêcheur. 


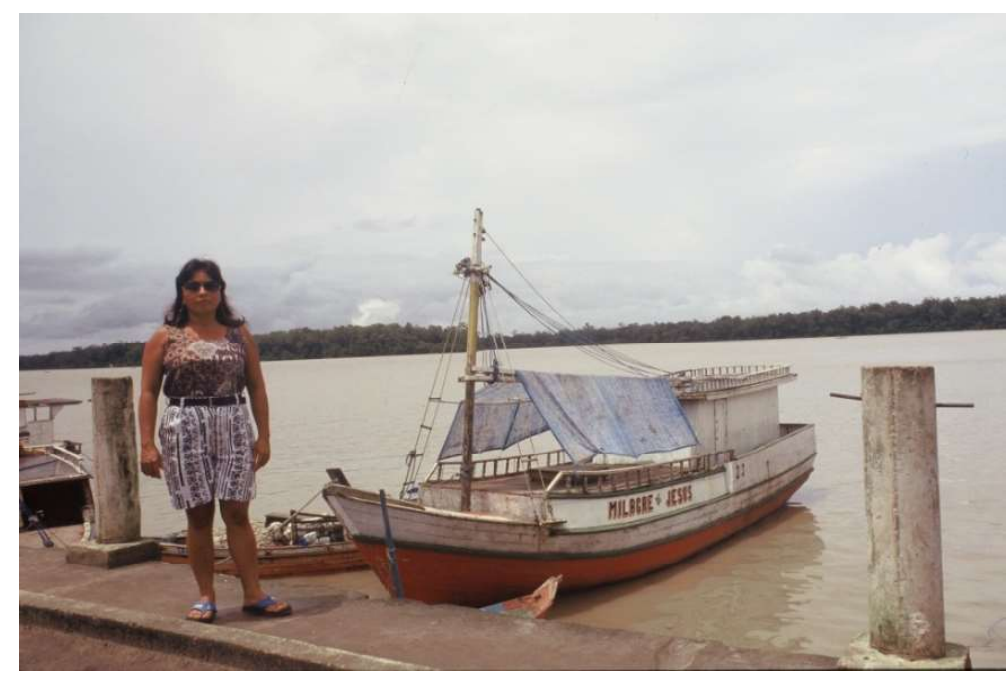

Fig. 9 - Ângela à Vigia devant son bateau

Après que les autorités policières eussent retrouvé l'embarcation, Ângela décida d'en devenir à son tour le patron. Son acquisition, disait-elle, avait exigé de son mari et d'elle des années de sacrifice. Elle ne pouvait donc se résoudre à le vendre et, n'ayant aucun revenu, en prendre les commandes était son seul espoir de survie. Contre l'avis même de sa famille, qui la poussait à s'en débarrasser, Ângela constitua un équipage, embarqua et sous la surveillance du chef de pêche, apprit à pêcher. Condamnée par sa famille, par la communauté de pêcheurs, elle le fut également par l'église :

«L La fermme au foyer, Ângela, a lâché sa cuisine, ôté ses jupes, s'est habillée de bermuda, s'est emplie de courage et est allée pêcher en haute mer depuis que son mari a été assassiné dans le bateau en pleine pêche par un membre de l'équipage. Angela a gagnéde l'argent, s'est acheté une maison, mais elle a été expulsée de l'église - Dieu Est Amour - pour avoir abandonnéle port de la robe. » (O Pescador 1996 : 5)

Mais l'histoire d’Ângela a connu des péripéties que ne relate pas la version écourtée du journal. Car la première grande difficulté avait été de trouver des hommes d'équipage en qui elle pouvait avoir confiance. Au cours d'une campagne, le premier équipage vendit la production dans un autre port, vola l'argent et abandonna le bateau loin de Vigia. Cinq chefs de pêche se succédèrent et profitèrent de la situation pour la voler. Puis son nouveau compagnon lui emprunta son filet pour pêcher sur sa propre embarcation et ne lui rendit jamais.

Le prêt de matériel est chose courante entre pêcheurs. L'opération est assortie d'une obligation pour l'emprunteur : remettre une part de sa pêche au propriétaire du matériel. Bien qu'Ângela acceptât les conditions d'un tel engagement, aucun homme de la communauté ne voulut lui prêter un filet ou une palangre. Au cours de l'enquête de terrain, j'ai pris fait et cause pour Ângela et tenté de l'aider à trouver du matériel. J'ai interrogé les pêcheurs, parfois même leur épouse, pensant que des femmes seraient plus sensibles aux problèmes d'une autre femme. Ils refusèrent. Les raisons invoquées étaient qu'ils venaient justement de prêter leur matériel à un autre pêcheur... Aucun d'entre eux ne m'a dit ouvertement qu'ils ne voulaient pas s'engager avec une femme mais, parlant des collègues, tous disaient : «C'est parce qu'ils ne veulent pas traiter avec une fermme. ». Unanimement les pêcheurs 
affirmaient qu> elle devait abandonner, vendre le bateau, la pêche $\mathrm{n}>$ étant pas un métier de femme. Cet exemple montre bien les limites de cette dichotomie genrée mer/terre, professionnel/domestique. Les hommes tolèrent que les femmes pratiquent de petites pêches dans la mesure où le produit est destiné à l>alimentation de la famille, qu'elles leur apportent une aide ponctuelle et qu'elles n'entrent pas en compétition avec leur métier. Aller un matin tirer quelques crabes pour le déjeuner, $\mathrm{n}>\mathrm{est}$ pas assimiler à $1>$ acte de pêcher. Seuls les hommes travaillent ; les femmes gèrent leur foyer.

Cette question des activités permises (tolérées...) ou interdites selon le lieu où elles s'effectuent, se confirment également quand il s'agit d'activités parallèles comme la construction et la réparation des filets, activité déterminante dans une région où le maillant domine. Pourtant les conditions de travail des tisseuses ne fait pas davantage l'objet de reconnaissance professionnelle de la part des armateurs qui font appel à leur savoir. Vigia est pourtant reconnue comme étant «la terre des tisseuses» (terra das tecedeiras, Maneschy \& Escallier 2002) mais les femmes des autres localités s'y emploient également.

Tisser les filets de pêche maintient les femmes à domicile et peuvent s'occuper en même temps des tâches domestiques. Elles tissent entre les repas, quand elles ont un instant de libre, la nuit pour celles qui ont déjà une activité dans la journée, comme par exemple après l'école pour les petites filles (Fig. 10). Les femmes tissent le coton, plus rarement le nylon, un matériau relativement récent. Les pêcheurs disent : «Les filets en coton, on peut les réparer sur le bateau ou à la maison mais pour ramender un filet en plastique, il faut être dans la rue. » (Coelho, pêcheur, Marudá). Ceci explique peut-être pourquoi rares sont celles à qui on enseigne à tisser le nylon ou à ramender les filets. Les femmes de Vigia, São Caetano et Marudá, sauf exception, ne savent pas ramender et ne cherchent pas à apprendre à le faire. Elles disent que réparer les filets « c'est beaucoup plus difficile que de les tisser », c'est pourquoi ce sont les hommes qui le font. En fait, il $s>$ agit bien là $\mathrm{d}>$ une division du travail qui se fait encore par l>espace et son occupation. La symbolique du dedans-féminin et du dehors-masculin (Fig. 11) se répètent et l'explication donnée par les femmes n'est là que pour entériner la reconnaissance de l'existence d'une tradition (Maneschy \& Escallier 2002).

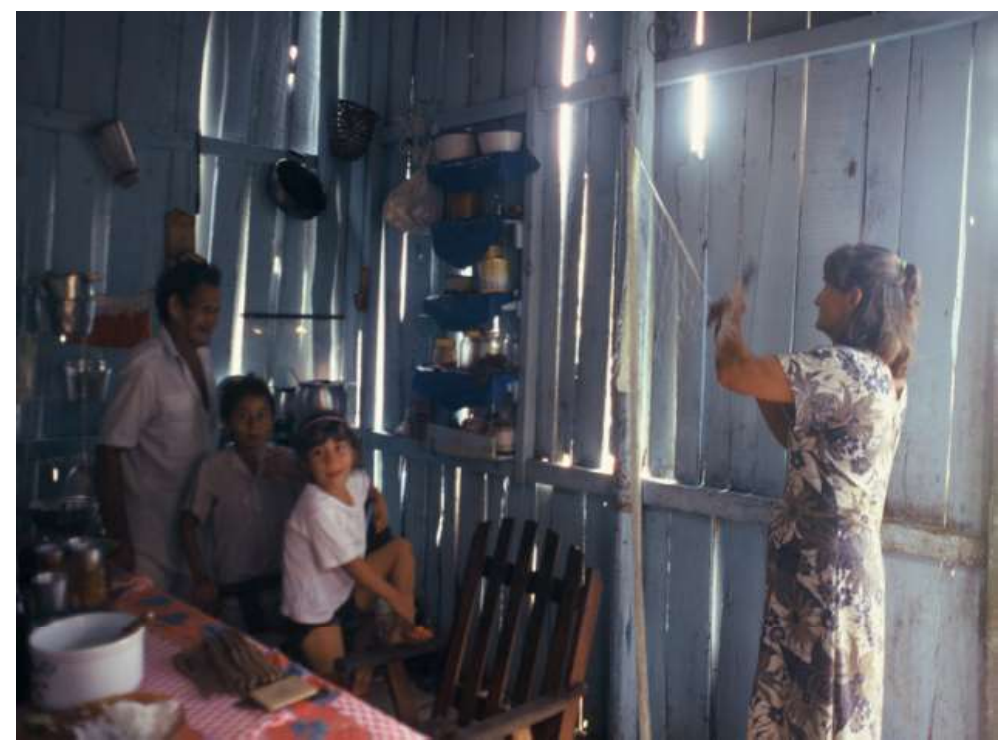

Fig. 10 - Femme tissant à la maison 


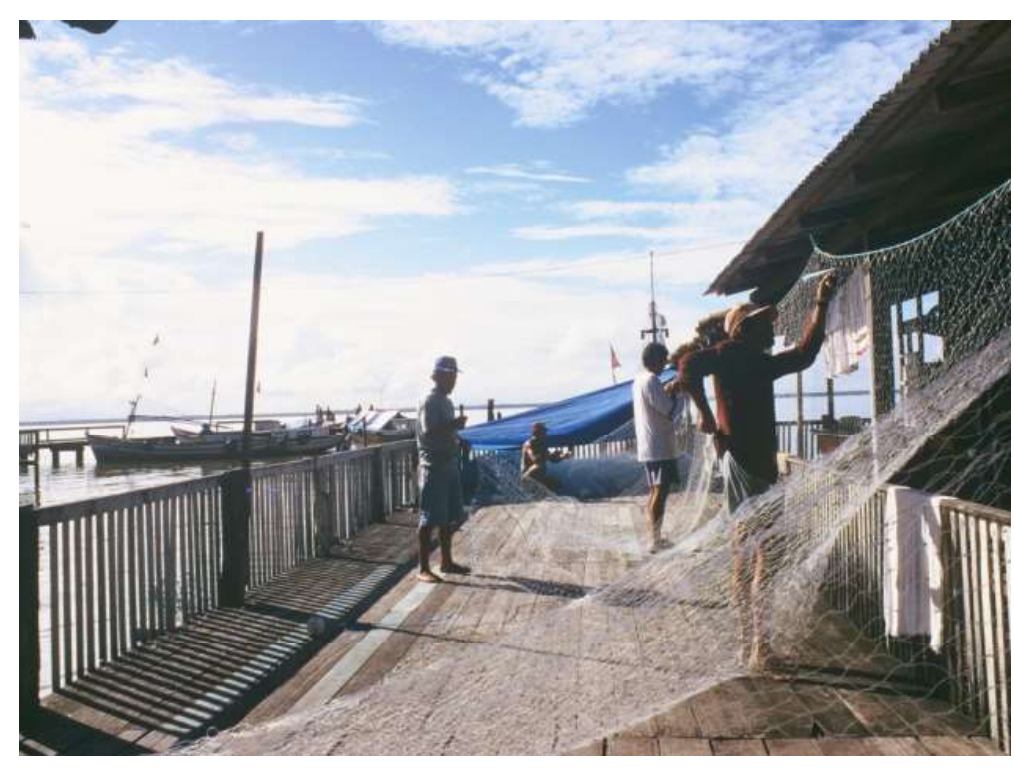

Fig. 11 - Hommes ramendant sur le port

Le tissage est donc féminin et le ramendage masculin. Les très rares cas que j’ai rencontrés, ou les rumeurs rapportées, de femmes sachant ramender et d'hommes tisser, ne permettent pas de remettre en question cette division sexuée du travail. II est cependant certain que des femmes aident leur mari à réparer les outils de pêche mais la plupart du temps elles effectuent ces travaux à l'ombre d'un hangar ou dans une cour, c'est-à-dire hors de la vue d'autrui. Il ne s'agit pas d'action professionnelle mais d'entraide entre conjoints, permettant de compenser le manque éventuel de main-d'œuvre masculine.

Tisseuses de mère en fille, les femmes disent apprendre à tisser par nécessité, rarement pour le plaisir. Le tissage est un moyen comme un autre - comme vendre des bouillies ou du charbon de bois - pour se procurer un revenu. Parce qu'elle s'effectue à domicile et s'insèrent au milieu des tâches quotidiennes, cette activité est perçue comme faisant partie de l'ensemble des occupations domestiques - tenir la maison, cuisiner, surveiller les enfants, entretenir le jardin potager, etc. D'ailleurs les tisseuses ne cherchent pas l'ouvrage. Elles restent chez elle et sont contactées par les armateurs. Elles sont payées «à la brasse» de filet tissé mais il s'agit de la brasse du demandeur et non de celles qui effectuent le travail et qui est en général bien plus petite. Le prix est de ce fait très variable et peut aller du simple au double. Les femmes qui refusent de travailler pour le plus bas prix sont rares même si certaines pensent qu'elles devraient se soutenir pour imposer leur prix et faire pression sur les acheteurs. Cependant, si les femmes d'une même famille s'entraident dans les périodes difficiles où l'argent et la nourriture viennent à manquer, celles-ci ne cherchent pas à s'unir avec leurs consœurs pour mieux se défendre contre les abus :

«Ici c'est diffcile, les gens s'entraident qu'au sein d'une même famille. On a déjà essayé de se réunir; pas tous les jours, de temps à autre seulement, mais on parle et on ne fait rien. » (Elisabeth, Vigia)

Cette absence de revendication de leurs droits tient au fait que les femmes ne sont pas toujours conscientes de détenir un savoir, un savoir-faire technique qui répond à une vraie demande et ce mal- 
gré l'introduction de filets de plastique sur le marché. Il est difficile de faire une estimation du nombre de tisseuses à Vigia dans la mesure où toutes les femmes disent avoir appris en regardant leurs aînées. Cette source de revenu, petite mais essentielle au maintien d'un équilibre économique, est cependant menacé par l'importation de filets chinois manufacturés, à très bas prix. Pourtant elles sont suffisamment nombreuses pour s'imposer et bien que la main-d'œuvre féminine soit très bon marché, leur expérience concurrence encore fortement la fabrication des filets industriels. On peut cependant s' interroger : quel impact aura l'introduction de nouvelle technologie sur l'emploi féminin ? Les conséquences seront-t-elles identiques à celles déjà observées dans d'autres régions du monde c'est-à-dire la disparition des tisseuses ou bien encore une plus grande exploitation de la main-d'œuvre féminine, là où sévit déjà le chômage.

Quoi qu'il en soit, qu'elles pêchent, tissent ou commercent, les femmes, véritable réservoir de main-d'œuvre à bon marché, tiennent une place importante dans l'économie communautaire. Les pêcheurs ont toujours pratiqué leur métier en impliquant fortement les femmes. Pourtant le caractère ambigu de la notion de «travail féminin» continue de se manifester par l'hésitation à reconnaître à celui-ci une existence autonome.

Ce constat impose des choix de société qui relèvent de la solidarité. C'est pourquoi il est essentiel d'inciter les femmes à se parler, communiquer et s'associer afin de défendre leurs droits. Dans le cadre du projet Women in Fishing ${ }^{18}$, j'ai organisé divers séminaires, notamment à Vigia réunissant plus d'une centaine de femmes venues de tous les villages avoisinants pour une journée de paroles, d'échanges, dans le but de leur permettre de partager leurs expériences et de créer des associations de défense. Au cours de ce séminaire, des femmes ont accepté de venir s'exprimer pour la première fois en public sur leurs conditions de vie (Escallier \& Maneschy 1996).

Pour l'anecdote, il convient de souligner que pour réunir tant de femmes qui, pour la plupart ne sont pas autorisées à sortir de chez elle (sauf le dimanche pour aller à la messe), il a fallu des mois de préparation, de «conditionnement» des intéressées : passer chaque semaine à leur domicile pour leur rappeler la date et l'importance de la rencontre ; leur distribuer des tracts ; de pourparlers avec leurs maris pour obtenir le droit de venir les chercher en van le jour dit $^{19}$; organiser une garderie pour les enfants qui ne pouvaient rester seuls, les pères refusant de s'en occuper. Passée la rumeur qui courait comme quoi cette organisation avait pour seul but de kidnapper les enfants... c'est avec la promesse qu'il leur serait servi un déjeuner «spécial», avec de la viande, si rare au menu du quotidien, que j’ai fini par convaincre leur mère !

18 En collaboration avec le projet RENAS du Musée Paraense Emilio Goeldi et Lourdes Furtado, et le Département des Sciences humaines de l'université de Belém-Universidade Federal do Pará et Cristina Maneschy, le collectif ICSF-International Collective in Support of Fishworkers (Inde) a mis en place le programme WIF-Women in fishing portant sur « le rôle et le statut des femmes de pêcheurs de la région du Nordeste paraense et zone de Salgado ». Ma mission auprès de ces institutions a été financée par le Ministère des Affaires étrangères français (bourse d'Excellence LAVOISIER)

En collaboration avec le projet RENAS du Musée Paraense Emilio Goeldi et le Département des Sciences humaines de l'Université de Belém (Universidade Federal do Pará)..

19 Deux vans avec chauffeurs, rencontrés sur le marché de Vigia, avaient été frétés par nos soins. Le jour de la réunion, ils devaient se présenter à 7 heures de matin afin d'effectuer avec moi un ramassage des participantes. Les deux chauffeurs ne furent pas au rendez-vous. Quelques jours plus tard, les rencontrant, je tentais d'obtenir une explication. Leurs bredouillements laissaient entendre qu'ils n'avaient aucune explication, et que le peu d'intérêt qu'ils portaient à l'événement - réunir des femmes pour la défense de leurs droits - ne méritait certainement pas de se lever si tôt ! 
Pour évaluer le rôle social qu'elles s'attribuaient, les participantes devaient décliner leur «métier». L'occupation la plus souvent déclarée était «femmes au foyer». Une femme sur deux déclarait avoir au moins deux activités sinon trois (agricultrice et pêcheuse, femme au foyer-agricultrice et pêcheuse, etc.) ; 55 femmes disaient pêcher régulièrement, mais seules 17 d'entre elles déclaraient être pêcheuses professionnelles (bien que sans cédula). Sur les 76 femmes de pêcheurs présentent, 40 pêchaient avec leur mari. Des 51 femmes sachant tisser les filets, seules trois d'entre elles se déclaraient tisseuses de profession, les autres s'estimant, avant tout, femmes au foyer et ne mentionnaient cette activité que si on les interrogeait. Deux femmes armatrices ont assisté à la réunion. Elles se sont d'abord inscrites comme «pêcheuse et femme au foyer» mais elles se sont ensuite clairement identifiées lorsqu'elles ont pris la parole en public. Et c'est d'ailleurs à cette occasion qu'Ângela prit la parole et que l'une des deux armatrices de Vigia, touchée par son récit, offrit de lui prêter un filet !

Moins visible, moins reconnu et moins valorisé que celui des hommes, le travail des femmes n'a toujours pas acquis sa pleine légitimité. Les femmes sont une catégorie sociale très docile mais pourtant très compétente. Sans statut, ou proche de celui de l'enfant, elles sont surexploitées, condamnées à un travail non reconnu, parcellisé, rarement rétribué ou sous-payé. Aussi il n’est pas nécessaire de leur donner une véritable formation professionnelle : soit elles exercent une activité non catégorisé excluant toute formation - la pêche de subsistance -, soit elles travaillent à domicile et leur formation se confond alors le plus souvent avec leur éducation familiale - le tissage. Cependant ces femmes sont aussi des épouses ou des concubines, des mères, c'est-à-dire un personnage central de la famille. Aussi se trouvent-t-elles prises dans une contradiction entre l'exploitation du travail féminin et la valorisation de la femme au foyer.

Cette intériorisation des modèles Homme-Femme/Homme-Milieu s'est révélée tout au long de l'enquête à la fois à travers les actes, les discours mais aussi les non-dits. Rares sont celles qui parlent de métier et qui pensent à s'inscrire dans un organisme professionnel comme la colónia, structure ayant pour mission d'enrôler les pêcheurs et de recueillir leurs cotisations pour le calcul des retraites. Il est vrai que les femmes-pêcheuses comme les tireuses de crabes rencontrent beaucoup d'hostilité, notamment de la part de présidents de colônias qui leur dénient le droit de s’y inscrire. Si en 1996, à Vigia, une vingtaine de femmes avait un passeport maritime, il avait été déterminé à l'époque, qu'il s'agissait d'une politique opportuniste menée par les élus locaux, des élections municipales se profilant à l'horizon. Sinon on leur refusait purement et simplement cette pièce administrative qui leur donnait accès aux droits inhérents aux professionnels de la pêche, et ceci à l'encontre des lois que les responsables interprétaient selon des conventions sociales sexistes. Ainsi à São Caetano de Odivelas, le responsable de la colônia a maintenu, durant des années, une position discriminatoire en évitant par tous moyens de communiquer aux femmes une copie des statuts régissant la profession. Plus tard, leur consultation prouvera, en effet, qu'il n'était fait mention d'aucun interdit concernant le sexe de l'adhérent et que celles qui pêchaient et récoltaient dans la mangrove pouvaient prétendre au bénéfice des avantages sociaux, comme les tireuses de crabes auxquelles on refusait un statut reconnu pourtant aux hommes exerçant cette même activité, dans les mêmes conditions techniques et spatiales. 
Les jeunes femmes de São Caetano m’ont alors investie d'une mission : me rendre à la colônia et demander à consulter les textes légaux afin de confirmer ce quelles supputaient : que la loi brésilienne ne faisait aucune distinction du genre concernant le statut de pêcheur. Il m’a fallu pas moins de quatre visites, le responsable feignant à chaque fois de ne pas savoir où le document se trouvait..., pour enfin en obtenir une copie, et seulement après avoir mentionnée que j'étais en mission sous l'égide d'un ministère français. Ce n'est donc pas la conscience professionnelle qui a fait agir ce fonctionnaire mais, dans le doute, la crainte de s'attirer d'éventuels ennuis.

\section{Les pièges de la discrimination}

Les conditions de vie, et de survie que les circonstances imposent aux femmes de pêcheurs, le poids des rapports du genre qui détermine les valeurs sociales et définit également les règles de la profession, contribuent à la transparence sociale des femmes. Sans statut, sans reconnaissance, sans retraite ou sécurité sociale, sans congés de maternité dans une société où les femmes ont de très nombreux enfants ${ }^{20}$, celles-ci sont parfois contraintes à des stratégies de survie sur lesquelles il est difficile de recueillir des témoignages. Cependant, et après plusieurs mois de terrain, je n'ai pu ignorer que certaines d'entre elles ont recours à la prostitution. Ainsi, les jours de marché, des femmes viennent s'offrir aux pêcheurs venus commercer. Elles se rendent au marché municipal ou sur le quai de débarquement et se donnent en échange d'un peu de poissons :

« Il faut aller dans la journée au marché municipal, là, les plus jeunes se vendent pourl $\mathrm{kg}$ de poisson. Ce sont les femmes des pêcheurs. » (Otávio, fonctionnaire de la côlonia, Vigia).

Des armateurs aussi profitent de leur position dominante d'employeurs et de créanciers pour tirer avantage des femmes venues quémander de l>argent, pour nourrir leur famille ou pour soigner un fils malade. De nombreux témoignages confirment que le chantage sexuel est exercé à l'encontre des plus jeunes femmes. Aux femmes jugées trop âgées ou sans attrait, ces hommes exigent à ce quelques repartent chez elles, et envoient à leur place l'une de leurs filles sous le fallacieux prétexte de venir chercher le prêt ou les provisions demandées à crédit. Certaines, contraintes, acceptent cette duperie et envoient leurs filles s'arranger avec le commerçant ou l'armateur usurier :

«Ça arrive que l>armateur profite de la situation quand la fermme $d>$ un pêcheur vient demander une seconde avance. Certains commerçants le font aussi et vont contraindre les plus jeunes.» (Rosa, 38 ans, São Caetano).

Il existe en effet un système de crédit permanant qui engage les pêcheurs et leur famille. Il est la conséquence des conditions drastiques dans lesquelles les hommes pratiquent le métier. Dans la région

20 En dehors du simple fait qu'elles n'ont pas toujours les moyens financiers de se procurer des contraceptifs oraux, des rumeurs circulent sur les préservatifs masculins comme quoi, oublié dans le corps d'une femme, celle-ci mourrait, et bien d'autres fables. Le nombre d'enfants par femme est très élevé ; souvent de pères différents. L'une de mes informatrices de São Caetano m'avait d'ailleurs offert de me donner son plus jeune fils pour l'emmener avec moi en France. 
du Salgado, l'embauche des hommes se fait sans contrat de travail. Le système de rémunération à la part ne garantissant pas un revenu fixe, les pêcheurs et leur famille vivent d'acomptes et de crédits. Quand les hommes embarquent, l'armateur avance aux épouses une somme qu'il estime équivalente à la moitié de la part que le pêcheur est sensé gagner. Pour une campagne de vingt et un jours, les épouses des fileyeurs reçoivent quelques dizaines de reais ${ }^{21}$. « Mon mari me laisse seule pendant 3 semaines avec 3 enfants. J'achète pour 20 reais de farine de manioc, du sucre, du café, du lait, un kilo de haricots, du riz. Je fais quelques réserves. Avec le reste, jachète un peu tous les jours des choses fraîches, du poisson, une poule... » (Vilma, 22 ans, Vigia)·Dans tous les cas étudiés, le solde du gain s'est avéré être toujours inférieur à la somme avancée. Quand l'avance est supérieure au gain, le pêcheur-débiteur se voit obligé de retravailler pour le même armateur. Il se retrouve alors pris dans un engrenage duquel il peut difficilement échapper.

Le système du crédit est une pratique généralisée dans les communautés de pêcheurs faute d'un revenu régulier. Qu'il s'agisse de pêche au filet, à la palangre et plus encore au curral, les apports quotidiens sont médiocres. Chaque jour les femmes de pêcheur doivent acheter les produits de base de l'alimentation familiale. Elles peuvent acquérir ces denrées à crédit dans une épicerie du village choisie par l'armateur. Les commerçants majorent alors leurs prix d'environ dix pour cent. D’autres vont jusquà vingt pour cent et s'entendent ensuite avec l'armateur pour se partager les bénéfices :

« Quand le pêcheur rentre, le patron de l'épicerie présente la note à l'armateur. Ils sont en cheville l'un et l'autre. Cette note est très élevée. L'armateur va alors déduire la somme due de la part revenant au pêcheur. Entre temps, il y a eu un accord entre les deux individus pour se répartir l'argent gagné. » (Otávio, São Caetano, fonctionnaire à IBAMA ${ }^{22}$ )

L'armateur récupère ainsi une partie du gain des pêcheurs qu'il emploie. Les pêcheurs et leurs épouses disent qu'ils sont «prisonniers», «esclaves» des armateurs, et des commerçants, car ils vivent selon un système qui les oblige en permanence.

Quelques années auparavant, des femmes venues des maisons closes de Vigia, où elles travaillent à l'abattage, ont commencé à embarquer, pour soi-disant faire la cuisine durant la campagne de pêche. Etant donnée la durée moyenne de trois semaines des sorties en mer, elles dormaient à bord.

«Au bordel, j'y étais l'autre samedi [...]. II y avait beaucoup de gens jusqu'à 4 heures du matin. J'ai vu une fermme monter avec 12 hommes cette nuit-là. En général les prostituées sortent vers minuit des bordels et vont vers les quais. Elles y rencontrent les pêcheurs et parfois trouvent un embarquement. Même si elles «travaillent»à bord en plus de faire la cuisine, c'est tout de même moins dur de voyager avec un équipage de 4 hommes que de travailler au bordel. » (Alfonço, Vigia, IBAMA)

21 A l'époque de l'enquête un real valait $0,58 €$; le salaire minimum brésilien étant de 100 réais/mois $(58 €)$. Aujourd'hui, le réal est à 0,28 et le salaire minimum autour 880 réais $(242 €)$.

22 Institut brésilien de l'environnement et des ressources naturelles renouvelables, organisme chargé de la protection de l'environnement au Brésil. 
Si ces prostituées professionnelles ne sont pas à proprement parler des épouses de pêcheurs, elles sont cependant leurs filles ou leurs nièces... De Vigia, ou des communautés halieutiques avoisinantes, elles travaillent en maison dans le même but : celui de subvenir aux besoins de la famille.

Cette affirmation de préférer l'embarquement à la maison close a été confirmée par d'autres témoignages, cette fois-ci féminins. Ils éclairent sur le sens pratique de la stratégie, reconnaissant sans détour «qu'il est moins fatiguant de satisfaire 4 ou 5 pêcheurs, bien las après une dure journée de pêche, plutôt qu'une douzaine à terre! » (Escallier 2011). Cependant le sort de ces femmes n'est pas plus enviable car elles sont à la merci des pêcheurs durant toute la marée ; de l'objet domestique déclaré, à l'objet sexuel sous-entendu, les femmes deviennent à bord des esclaves qui n'ont d'autre recourt contre la maltraitance que d'attendre le retour à terre de l'équipage et de retourner à la misère du quotidien.

Quand il n'y a ni travail, ni emploi, que durant des mois aucun armateur ne vient frapper à la porte d'une tisseuse de filets pour lui donner de l'ouvrage, que les maris débarquent chaque fois plus endettés et dépendant de leur patron, se vendre pour un peu de poissons, accepter les avances d'un débiteur, ou devenir pensionnaire d'une maison close sont des stratégies, d'autres stratégies de survie...

\section{Conclusion}

Catégorisant les femmes en un groupe social inférieur, les hommes sont aussi les perdant d'un système de pensée qui les obligent autant que les femmes à reproduire un mode d'organisation et de distribution des tâches sexuées en fonction d'une typologie spatiale identifiant généralement la femme au-dedans-le rivage et l'homme au dehors-la mer. Les conditions de vie des femmes, ainsi que l'avenir des enfants, garçons et filles, déjà très engagé, devraient dans la logique permettre aux femmes et aux associations qu'elles ont créées dans les années qui ont suivi cette étude de terrain ${ }^{23}$, d'exiger le droit de travailler dans le respect de la loi et l'élimination de toute discrimination. Car que restera-t-il aux tisseuses quand les filets chinois, prêt à l'emploi, viendront remplacer les filets de coton ? La pêche ! Les pêcheurs doivent en prendre conscience et comprendre que nier le droit des femmes, c'est œuvrer contre leurs propres intérêts et ceux de leur communauté.

La reconnaissance juridique des pêcheuses est nécessaire pour assurer l'accès aux prestations sociales et de sécurité du travail, y compris la réalisation des principes constitutionnels d'égalité entre les hommes et les fermmes, et la dignité du travail. $\gg^{24}$ (Boshi et al. 2012)

On note que chaque fois qu’une communauté, une société, vient à revendiquer des droits aux femmes, les conséquences tant politiques, économiques que sociales ont toujours eu des retombées positives sur les conditions de travail des hommes, une logique répétée à l'envi notamment par la Com-

23 Des associations de fileuses ont été créées au Pará. Dans le même objectif, et sous l'égide de AKTEA, diverses associations ont été montées en Europe.

24 "El reconocimiento legal de las pescadoras es necesario para asegurar el acceso a las prestaciones de la Seguridad Social y Laboral, incluso por la realización de los principios constitucionales de igualdad entre hombres y mujeres, y la dignidad del trabajo." 
mission des Pêches du Parlement européen. Quand les femmes revendiquent des droits sociaux tel qu'un statut légal reconnaissant leur contribution au sein des entreprises de pêche, leurs actions souvent aboutissent à défendre l'outil de travail et donc la survie du secteur de la pêche, des entreprises et des communautés de pêche dans leur totalité (Frangoudes 2014).

Les droits des femmes de pêcheur - ou plus justement des pêcheuses - est la question centrale qui m'a amenée à participer au Programme Womem in Fishing in Brazil de l'ICSF. Cette étude a donné une place importante à la parole des femmes. Elle a permis de dire le non-dit, de voir le non-visible à partir d'une approche ethnologique des discours et des actes permettant une meilleure perception des représentations sociales. Il en a découlé la constitution d'associations statutaires, dont huit à Vigia recensées en $2007^{25}$, permettant de mieux structurer les activités féminines en général et plus spécifiquement celles des pêcheuses.

Les pêcheuses du Pará ne se sont pas assises sur le rivage du monde masculin ${ }^{26}$. Elles se sont engagées sur un chemin de desserrement du carcan social, telles leurs consœurs par-delà les mers et les océans ${ }^{27}$.

\section{Christine Escallier é maître de conférences na Universidade da Madeira e pesquisadora associada do Centro em Rede de Investigação em Antropologia (CRIA), em Lisboa.}

25 AMAPESCA-Associação das Mulheres Pescadoras da Comunidade de Vigia, créée dans le but de confectionner et commercialiser les filets de pêche ; ASMUPEB-Associação das Mulheres Pesqueiras das Regiões das Barretas, pour commercialiser la chair de crabe.

26 Expression inspirée par le titre de la pièce Nous étions assis sur le Rivage du monde (José Pliya 2004)

27 La création d'associations de femmes de pêcheur est une stratégie développée par l'Union européenne qui finance divers programmes. J'ai été responsable scientifique du Réseau Thématique FEMMES-Portugal/Qualité de la vie et gestion des ressources du vivant (4e Programme-Cadre pour la Recherche et le Développement technologique-PCRD - Projet n ${ }^{\circ}$ Q5TN-2002-01560 CCE, DG Pesca 5 et Programme QLK5-2001-01560). A cet effet est né le Réseau Européen des organisations de femmes de la pêche et de l'aquaculture-AKTEA. http://akteaplatform. eu/?lang=fr Ses objectifs étaient de rendre visible le rôle des femmes dans la pêche et les activités connexes ; faire connaître la contribution des femmes dans ces secteurs ; promouvoir la participation des femmes dans les processus de décision au sein de ces secteurs ; etc. Le réseau est à l'origine de la constitution d'organisations de femmes en Grèce, Italie, Portugal, Grande-Bretagne et a regroupé une douzaine d'associations déjà existantes (Espagne, France, Pays-Bas). 


\section{BIBLIOGRAPHIE}

ALENCAR, Carlos Alexandre Gomes de. 2014. Pesca epobreza no Brasil. Ph.D. Dissertation. Fortaleza, CE: Universidade Federal do Ceará.

ALENCAR, Edna Ferreira. 1991. Pescadeiras, Companheiras e Perigosas. Um Estudo sobre a Pesca Feminina em Lençóis. Brasília: UnB.

ALENCAR, Edna Ferreira. 1993, "Gênero e Trabalho nas Sociedades Pesqueiras", In: L. Furtado, W. Leitão \& A.F. de Mello (eds.). Povos das Águas, Realidade e Perspectivas na Amazônia. Belém: MCT/ CNPq//Museu Goeldi: 63-81.

ALENCAR, Edna Ferreira. 1997. Trabalho feminino e participação política como instrumentos para a manutenção e renovação da qualidade de vida. In: Lourdes Gonçalves Furtado. (org.). ?Amazônia, Desenvolvimento, Sociodiversidade e Qualidade de Vida? Belém: Universidade Federal do Pará.

BECK, Ana Maria. 1979. Lavradores e pescadores: um estudo sobre o trabalho familiar e trabalho acessório. Dissertação de Mestrado, UFSC.

BOSCHI AGUIAR DE OLIVEIRA, Olga Maria \& Vera Lucia Da SILVA. 2012. ¿Pescadoras o “mujeres de pescador"? La regulación jurídica de la pesca artesanal en Brasil y el problema de no reconocimiento del trabajo profesional de las pescadoras en Santa Catarina. Investigación y género, inseparables en el presente y en el futuro, 1(1): 1327-1352.

DIEGUES, Antônio Carlos. 1999. "A sócio-antropologia das comunidades de pescadores marítimos no Brasil”. Etnográfica 3 (2): 361-375.

DIRENBERGER, Lucia \& Camille SCHMOLL. 2014. « Ce que le genre fait à l'espace... et inversement ». In: L. Direnberger \& C. Schmoll, Le tournant spatial dans les études du genre. Les cahiers du CEDREF 21.

ESCALLIER, Christine. 2011. «A prostituição como estratégia de sobrevivência nas comunidades piscatórias do Pará-Brasil », Anais Electrônicos do XI Congresso Luso-Afro-Brasileiro de Ciências Sociais, Bahia, Salvador: 7.

ESCALLIER, Christine. 2013. Women's networks and Associations / Réseaux et Associations de Femmes en Europe. Audition Publique du 10 octobre 2013, Parlement Européen, Bruxelles. Vidéo http://www.europarl.europa.eu/ep-live/fr/committees/video?event=20131014-1600-COMMITTEE-FEMM-PECH

ESCALLIER, Christine. 2014. Les pêcheurs de Nazaré (Portugal). L'empreinte de la mer. Paris: L?Harmattan. ISBN: 978-2-343-03178-1.

ESCALLIER, Christine \& Maria Cristina MANESCHY. 1996. « Encontro de mulheres na pesca reuniu mais de 100 mulheres em Vigia », Boletim Taras, GEPEM, UFEP. Belém-Brasil, 1 (4): 5-20. FAO. 2002. Problèmes auxquels sont confrontés pêcheurs et aquaculteurs. http://www.fao.org/docrep/pdf/005/y7300f/y7300f02.pdf

FAO. 2003. Stratégies relatives au renforcement de la contribution durable de la pêche artisanale à la 
sécurité alimentaire et à la lutte contre la pauvreté. Comité de Pêches, Rome 24-28 février 2003. FAO. 2017. Rapport de la trente-deuxième session du Comité des pêches. Rome, FAO. ISBN 978-92-5209662-7-

FRANGOUDES, Katia. 2014. Women in fisheries: a European perspective. Brussels: European Parliament. ISBN: 978-92-823-4689-1.

FURTADO, Lourdes Gonçalves. 1987. Curralistas e Redeiros de Marudá: Pescadores do litoral do Pará. Belém, Museu Emilio Goeldi.

FURTADO, Lourdes Gonçalves. 1993. Povos das águas: realidade e perspectivas na Amazônia, PR/ MCT/CNPq-Museu Emílio Goeldi, Belem.

GEISTDOERFER, Aliette, Isabelle LEBLIC \& Jacqueline MATRAS-GUIN. 1992. « Statuts et fonctions des femmes dans les communautés maritimes et fluviales ». Anthropologie Maritime, cahier $\mathrm{n}^{\circ}$ 4, Paris : CETMA.

GODELIER, Maurice. 1982. La production des Grands Hommes. Paris: Fayard.

HALL, Edward T. 1971. La Dimension cachée. Paris: Seuil.

LEFEBVRE, Henri. 1974. La production de l'espace. Paris: Anthropos.

MANESCHY, Maria Cristina. 1995. A mulher está se afastando da pesca? Continuidade e mudança do papel da mulher na manutenção doméstica entre família de pescadores no litoral do Pará. Belém: Museu Paraense Emílio Goeldi 11(2): 145-166.

MAMESCHY, Maria Cristina \& Christine ESCALLIER. 2002. «Parceiras de terra: o trabalho das mulheres na pesca em Vigia, litoral do Pará », In: L. Furtado \& H. Quaresma (dir.), Gente e ambiente no mundo da pesca artesanal. Belém: Museu Paraense Emílio Goeldi: 55-89.

MOTTA-MAUÉS, Maria Angelica. 1993. "Trabalhadeiras” \& "Camarados": Relações de Gênero, Simbolismo e Ritualização numa Comunidade Amazônica. Belém, UFPa.

MOTTA-MAUÉS, Maria Angelica. 1999. Pesca de homem/peixe de mulher (?): Repensando gênero na literatura acadêmica sobre Comunidades Pesqueiras no Brasil. Etnográfica, 3 (2): 377-399.

PLIYA, José. 2004. Nous étions assis sur le Rivage du monde... Paris : L’Avant-Scène, Col. Quatre-Vents.

WOORTMANN, Ellen F. 1992. "Da Complementaridade à Dependência: Espaço, Tempo e Gênero em Comunidades 'Pesqueiras”. Revista Brasileira de Ciências Sociais, 18: 41-61. 


\title{
MER, RIVIÈRE, MANGROVE : LES ESPACES MARQUÉS PAR LE GENRE DANS LES COMMUNAUTÉS DE PÊCHE DU PARÁ, BRÉSIL
}

Résumé : La représentation sexuée de la mer transparaît dans de nombreux récits - tant mythologiques que poétiques - qui décrivent le rivage comme un lieu d'attente où la femme espère le retour du marin - explorateur ou corsaire - déduisant ainsi que les mers et les océans sont avant tout des espaces masculins. En réalité, et dans de très nombreuses communautés de pêcheurs, les femmes exploitent les eaux qui les environnent. Elles sont pêcheuses. Or, ce n'est pas l'activité mais principalement la qualité des eaux exploitées - douces ou salées -, et le mode d'accès aux pêcheries - avec ou sans navigation - qui déterminent le statut social et professionnel des femmes des zones mangroviennes du Brésil. Le poids des traditions les maintient dans cette imagerie populaire de l'espace marin interdit contre laquelle elles luttent pour une reconnaissance de leurs droits d'exercer une profession sans limite spatiale, technique ou sociale.

Cet article, basé sur une enquête de terrain réalisée dans une quinzaine de villages estuariens et fluviaux le long du furo Santa Maria, voie de communication naturelle entre l'Amazone et la baie de Marajó, propose une lecture anthropologique des espaces de pêches en y introduisant la notion de genre, et ses conséquences sur les représentations et les pratiques sociales et économiques paraenses.

Mots-clefs : espace genré, pêche artisanale, igarapé, mangrove, Brésil

\section{SEA, RIVER, MANGROVE. THE SPACES MARKED BY THE GENDER IN THE FISHING COMMUNITIES OF THE STATE OF PARÁ, BRAZIL}

\begin{abstract}
The sexual representation of the sea is reflected in many stories - both mythological as poetic - that describe the shore as a place of waiting where the woman hopes the return of the sailor - explorer or privateer - deducting and the seas and the oceans are above all male spaces. In fact, and in very many communities of fishermen, women operate the waters that surround them. They are fisherwomen. However, this isn't activity but mainly the exploited - fresh or salt water - and the mode of access to fisheries - with or without navigation - that determine the social and professional women's status in the Brazil tropical swamp. The weight of traditions maintains them in this popular imagery of prohibited marine space against which they are fighting for recognition of their rights to exercise a profession without space, technical or social limits. This article, based on a fieldwork conducted in 15 estuarine and river village along the furo Santa Maria, natural communication channel between the Amazon and the Bay of Marajó, proposes a reading of anthropological fishing spaces by introducing the notion of gender, and its consequences on representations and social practices and economic paraenses.
\end{abstract}

Keywords: gender area, artisanal fishing, igarapé, mangrove, Brazil

RECEBIDO: $17 / 02 / 2018$

APROVADO: $05 / 11 / 2018$ 
162 CAMPos V.19 N.1 jan.jun.2018 